\title{
In-fiber Long-period Grating and Fiber Bragg Grating-based Sensor for Simultaneously Monitoring Remote Temperature and Stress
}

\author{
Hung-Ying Chang, ${ }^{1}$ Chien-Hung Yeh, ${ }^{2}$ Chuan-Ying Huang, ${ }^{1}$ \\ Ming-Yue $\mathrm{Fu}^{3,}{ }^{3 *}$ Chi-Wai Chow, ${ }^{4}$ and Wen-Fung Liu ${ }^{5}$ \\ ${ }^{1}$ Electrical and Communications Engineering, Feng Chia University, Taichung 40724, Taiwan \\ ${ }^{2}$ Department of Photonics, Feng Chia University, Taichung 40724, Taiwan \\ ${ }^{3}$ Center for General Education, Air Force Academy, Kaohsiung 82047, Taiwan \\ ${ }^{4}$ Department of Photonics and Institute of Electro-Optical Engineering, \\ National Chiao Tung University, Hsinchu 30010, Taiwan \\ ${ }^{5}$ Department of Electrical Engineering, Feng Chia University, Taichung 40724, Taiwan
}

(Received November 9, 2016; accepted June 13, 2017)

Keywords: fiber sensor, fiber Bragg grating, long-period grating, superstructure fiber Bragg grating

In this study, we experimentally demonstrated that a high-sensitivity fiber sensor can be used for the remote sensing of temperature and stress simultaneously. The proposed sensor includes several sensing heads, each of which is composed of a long-period grating (LPG) and a fiber Bragg grating (FBG). Moreover, the optical characteristics of both the FBG and LPG are different. On the basis of these different optical features and the sensing transfer matrix, temperature and stress can both be remotely measured simultaneously. The temperature sensitivities of the grating-wavelength shift and grating-reflection intensity are about $0.0082 \mathrm{~nm} /{ }^{\circ} \mathrm{C}$ and $0.044 \mathrm{dBm} /{ }^{\circ} \mathrm{C}$, respectively. The strain sensitivities of the gratingwavelength shift and grating-reflection intensity are respectively around $0.0133 \mathrm{~nm} / \mathrm{g}$ and 0.0089 $\mathrm{dBm} / \mathrm{g}$. Therefore, the fiber sensor can provide a simple method with excellent sensitivity for applications of structure-security monitoring and industrial engineering measurements.

\section{Introduction}

There are many different conventional methods for measuring temperature or stress using electrical, ultrasonic, mechanical, and optical techniques. Among the conventional methods, the common electrical types of stress or temperature sensors have already been commercialized. In general, for many practical measuring environments, temperature and stress have correlation dependence. Thus, in this case, the sensor should be designed to have the ability to simultaneously and remotely monitor temperature and stress to demodulate the two parameters. However, the sensors used in conventional methods require more complex designs for simultaneously sensing two different physical parameters. For fiber sensors, a series of sensing techniques have already been proposed to overcome these restrictions, such as hybrid

*Corresponding author: e-mail: fumy1109@yahoo.com.tw

http://dx.doi.org/10.18494/SAM.2018.1502 
fiber Bragg grating (FBG)/long-period grating (LPG). Patrick et al. used a series of LPGs to connect FBGs to simultaneously sense temperature and strain with a measurement range of \pm 9 $\mu$ strain and $\pm 1.5^{\circ} \mathrm{C}^{(1)}$ For a dual-wavelength fiber grating sensor, $\mathrm{Xu}$ et al. used two gratings with different Bragg wavelengths to simultaneously measure temperature and strain. ${ }^{(2)}$ Based on a FBG cavity sensor, Du et al. used a fiber grating Fabry-Perot cavity structure to monitor temperature and strain with a measuring range of 0-3000 $\mu$ strain and $20-60{ }^{\circ} \mathrm{C} .{ }^{(3)}$ However, for detecting remote temperature and stress variations simultaneously, all the above-mentioned techniques or conventional methods have problems of inaccuracy and low sensitivity.

In this paper, we propose a new fiber sensor based on several sensing heads. Each sensing head includes an LPG concatenated with an FBG for simultaneously monitoring remote temperature and stress. Moreover, the proposed sensing mechanism is attributed to the different optical characteristics of both FBG and LPG. Hence, temperature and stress can be determined simultaneously by measuring the change in the reflective wavelength and reflective power of FBG.

\section{Theory}

In this study, the sensing head including an LPG cascaded with an FBG (FBG-LPG) is shown in Fig. 1 with the sensing optical characteristics of both the LPG and FBG.

The LPG is one of the fiber passive components in which the core index is periodically modulated along the axial direction of the fiber with a grating period of about several hundred micrometers. When the grating phase matching condition is satisfied, the light from the forward fundamental core mode is coupled into the cladding modes to cause several loss dips in the grating transmission spectrum [as shown in the transmitted spectrum (I) of Fig. 1] as described by the following equation: ${ }^{(6)}$

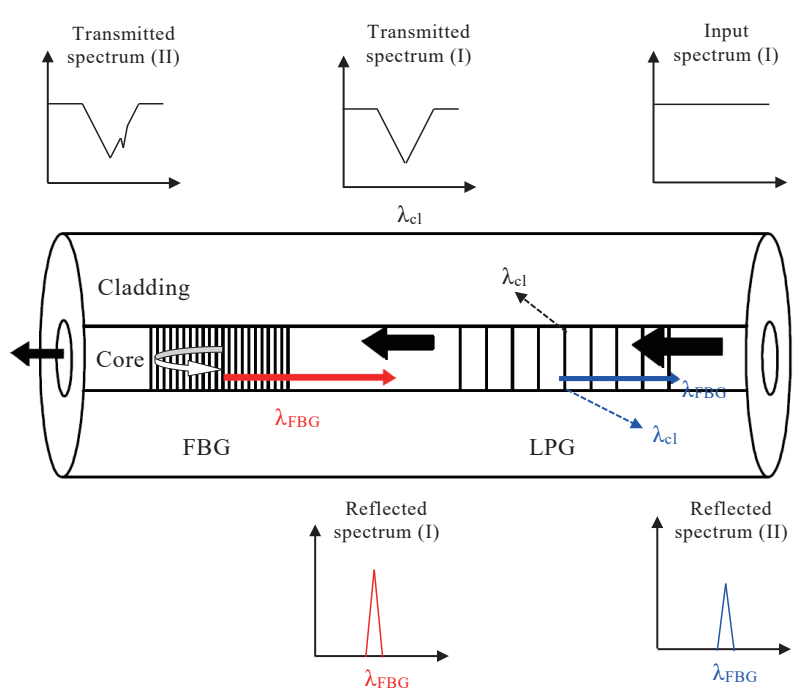

Fig. 1. (Color online) Schematic diagram of an FBG connected in series with an LPG for mode coupling. 


$$
\lambda_{c l}^{(m)}=\left(n_{c o}-n_{c l}^{(m)}\right) \Lambda_{L P G}
$$

where $\lambda_{c l}^{(m)}$ is the $m$ th order coupling wavelength, $n_{c o}$ is the effective index of the fundamental core mode, and $n_{c l}^{(m)}$ is the effective index of the $m$ th order cladding mode. $\Lambda_{L P G}$ represents the period of LPG. This fact implies that the LPG's resonant wavelengths are sensitive to the variation of the local environmental index surrounding an LPG.

FBGs are called short-period gratings and have a period of less than $1 \mu \mathrm{m}$. In general, the coupled-mode theory is often used to analyze the spectral characteristics of fiber gratings owing to its simplicity and accuracy. The relationship between the Bragg wavelength and the grating period is

$$
\lambda_{B}=2 n_{c o} \Lambda_{F B G},
$$

where $\lambda_{B}$ denotes the Bragg wavelength and $\Lambda_{F B G}$ represents the period of the FBG. From the coupled-mode theory and by substituting the boundary conditions of the fiber gratings into the wave equation, the maximum reflectivity of FBGs can be derived as $R_{\max }=\tanh ^{2}(\kappa l)$, where $\kappa$ and $l$, respectively, denote the coupling coefficient and total length of the fiber gratings. For the FBG fabrication, the phase mask writing technique is commonly utilized owing to the advantages of good repeatability and simplicity. In this study, the reflected wavelength of FBG [reflected spectrum (I) shown in Fig. 1] was fabricated on the positive hypotenuse as one of several loss dips of LPG [transmitted spectrum (II) shown in Fig. 1]. The reflected power of FBG is coupled into the cladding by the LPG, so the reflected power decreases as shown in reflected spectrum (II) of Fig. 1. Changing both the stress and temperature causes the variation of two grating parameters, namely, the first parameter is the resonant wavelength of LPG, resulting in the reflection power change of the FBG, and the second parameter is the reflection wavelength shift of the FBG.

The relationship between the shift of Bragg wavelength $(\Delta \lambda)$ with strain change $(\Delta \varepsilon)$ and temperature change $(\Delta T)$ can be expressed as ${ }^{(7)}$

$$
\left.\Delta \lambda=\lambda_{B}\left[\left(1-P_{e}\right) \Delta \varepsilon+(\eta+\xi) \Delta T\right)\right]
$$

where $P_{e}=\frac{n_{c o}^{2}\left[P_{12}-v\left(P_{11}+P_{12}\right)\right]}{2}$ denotes the strain-optic coefficient of the fiber, $v$ represents the Poisson ratio, $P_{11}$ and $P_{12}$ are the Pockel's coefficient components of the stress-optic tensor, $\eta$ is the thermal expansion coefficient of the fiber, and $\xi$ denotes the thermo-optic coefficient of the fiber. In addition, the relation between the grating reflectivity power change $(\Delta P)$ in Bragg wavelength with strain and temperature can be expressed as ${ }^{(7,8)}$

$$
\Delta P=\frac{m \pi I n_{d}}{2 \lambda_{B} n_{c o}}\left[\left(n_{d} \Lambda_{L P G}-2 P_{e}\right) \Delta \varepsilon+\left(n_{d} \eta+2 \xi\right) \Delta T\right]
$$

where $m$ is the total period number of the LPG, $I$ is the overlap integral area of fiber between 
the core mode and cladding mode, and $n_{d}$ is the index difference between the core and cladding index.

When stress is applied to a sensor with a cross-sectional area of the fiber a and with an elasticity coefficient of $E_{f i b e r}$, the relationship between the stress and the strain is

$$
\Delta \varepsilon=\frac{-v \Delta S}{a E_{\text {fiber }}}
$$

By combining Eqs. (3)-(5), both the Bragg wavelength shift and the reflectivity power variation of an LPG concatenated with an FBG related to various stress or temperatures can be expressed as

$$
\begin{gathered}
\Delta \lambda=\lambda_{B}\left(1-P_{e}\right) \frac{-v \Delta S}{a E_{\text {fiber }}}+\lambda_{B}(\eta+\xi) \Delta T, \\
\Delta P=\frac{m \pi I n_{d}}{2 \lambda_{B} n_{c o}}\left(n_{d} \Lambda_{L P G}-2 P_{e}\right) \frac{-v \Delta S}{a E_{\text {fiber }}}+\frac{m \pi I n_{d}}{2 \lambda_{B} n_{c o}}\left(n_{d} \eta+2 \xi\right) \Delta T .
\end{gathered}
$$

From Eqs. (6) and (7), we can obviously see that the fiber gratings fabricated using different types of fibers have different wavelength shifts and the reflectivity changes of the narrowband channels versus various stresses and temperatures. This is due to the fact that the spectral characteristics for FBG and LPG are different. Moreover, Eqs. (6) and (7) can be simplified to Eq. (8), by which both stress and temperature can be simultaneously obtained by experimentally measuring the shift of the center wavelength $(\Delta \lambda)$ and the reflectivity power change $(\Delta P)$ in one of the narrow-band loss dips.

$$
\begin{aligned}
& \Delta \lambda=M_{11} \Delta S+M_{12} \Delta T \\
& \Delta P=M_{21} \Delta S+M_{22} \Delta T
\end{aligned}
$$

Here, $\quad M_{11}=\lambda_{B}\left(1-P_{e}\right) \frac{-v}{a E_{\text {fiber }}}, \quad M_{12}=\lambda_{B}(\eta+\xi), \quad M_{21}=\frac{m \pi I n_{d}}{2 \lambda_{B} n_{c o}}\left(n_{d} \Lambda_{L P G}-2 P_{e}\right) \frac{-v}{a E_{\text {fiber }}}$, and $M_{22}=\frac{m \pi I n_{d}}{2 \lambda_{B} n_{c o}}\left(n_{d} \eta+2 \xi\right)$.

Thus, for the given coefficients of $M_{11}, M_{12}, M_{21}$, and $M_{22}$, the stress and temperature changes can be determined by solving the Eq. (8). In this sensing system, we can observe the reflection power change and grating wavelength variation to obtain stress and temperature simultaneously.

\section{Fiber Grating Fabrication}

In this study, the fiber grating was fabricated using a SMF-28 fiber and a UV beam from a $\mathrm{KrF}$ excimer laser $(248 \mathrm{~nm})$ passing through an amplitude mask for LPG and a phase mask 
for FBG. The fiber core diameter is around $9.2 \mu \mathrm{m}$ and the cladding diameter is about $125 \mu \mathrm{m}$ for the SMF-28 fiber from Fujikura Corp. In order to increase fiber photosensitivity for rapid grating growth, the SMF-28 fiber is soaked in hydrogen for two weeks. When the UV beam passes through a phase mask, the UV light is phase-modulated spatially and diffracted to form an interference pattern laterally and along the incident laser beam direction. The interference pattern creates a refractive index modulation in the photosensitive fiber along the fiber axis direction and to form the FBG. For the LPG fabrication, when the UV beam passes through an amplitude mask which doesn't produce diffraction fringes, the amplitude mask shades the laser light to form bright and dark fringes in fiber. By using different periods of amplitude masks, the required grating wavelength can be fabricated. The required grating reflectivity can be obtained by controlling the time of UV exposures. For this experiment, the FBG periods both of head 1 and head 2 are 535.36 and $536.79 \mathrm{~nm}$, respectively, and the LPG period of head 1 with $400 \mu \mathrm{m}$ is identical to that of head 2 .

\section{Experimental Setup}

Figure 2 shows the proposed experimental setup for simultaneously monitoring temperature and stress remotely, which includes a light-wave measurement system (8164A, Agilent), a tunable laser (81640A, Agilent), a power meter (81634A, Agilent), an in-line polarizer, an optical circulator (OC), a 50-km-long single-mode fiber (SMF), and two proposed sensing heads (Sensors 1 and 2). The sensing head is fabricated using an LPG concatenated with an FBG. The length of the two fiber gratings is about $20 \mathrm{~mm}$. The separation between the LPG and FBG is around $10 \mathrm{~mm}$. The tunable laser acting as a measuring light source has a minimum output power of $-13 \mathrm{dBm}$ and an operating range from 1510 to $1640 \mathrm{~nm}$ with a wavelength resolution of $0.1 \mathrm{pm}$. In this experiment, a computer is used to control the light wave measurement system

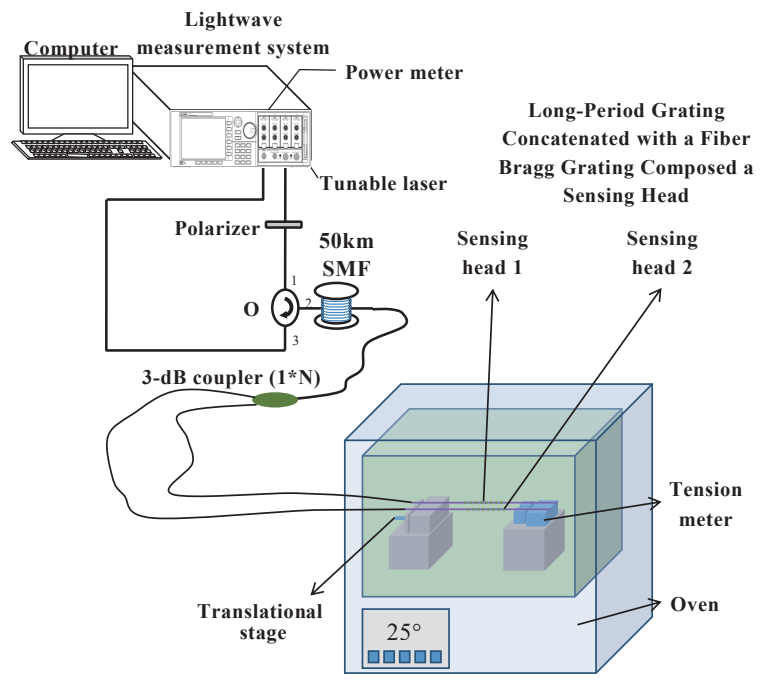

Fig. 2. (Color online) Experimental setup for monitoring temperature and stress remotely. 
to scan a range for the reflected wavelength of sensing heads. The reflected wavelength and power can be simultaneously measured to obtain the stress and temperature parameters. The polarizer inside the ring cavity ensures that the laser operates at the single-polarization state. By reducing the polarization-dependent loss (PDL), mode hopping is suppressed. An OC ensures the wavelength propagation in the clockwise direction. The experimental setup for the measurement both of the temperature variation and the strain tests is shown in Fig. 2. From this figure we can see that a translational stage, a tension meter, and two sensing heads are put inside the oven for the measurement of temperature and stress variation. The translational stage is used for changing the stress of the sensing head, and the tension meter is used for monitoring the stress value. One end of the sensing head is fixed on the translational stage and the other end is fixed on the tension meter. For the experimental measurement, the grating wavelength shift and reflection power variation can be monitored by an optical spectrum analyzer (OSA). In this experiment, two sensing heads are connected in parallel with reflection Bragg wavelengths of 1546.58 and $1552.65 \mathrm{~nm}$ for Sensors 1 and 2, respectively. For the LPG's loss dip wavelength, 1539.13 and $1547.23 \mathrm{~nm}$ are used for Sensors 1 and 2, respectively. Both the reflection Bragg wavelength and reflection power are affected by stress and temperature simultaneously. This system can demodulate the stress and temperature of test samples.

\section{Experimental Results}

\subsection{Temperature}

In the experimental measurement, the remote temperature can be obtained using two sensing heads at two different measuring points with different reflected Bragg wavelengths and reflected powers, as shown in Fig. 3(a). When the temperature is increased, the wavelength of FBG and the loss dip of LPG shifts toward longer wavelengths due to the thermo-optic effect and the thermal-expansion effect resulting in an increment of the grating effective refractive index and grating period. Because the Bragg wavelength is located on the negative bevel edge of the loss dip of LPG, the reflection power of FBG increases. The corresponding peak wavelength shift and the reflected power change are simultaneously monitored at a wavelength of $1546.58 \mathrm{~nm}$ by Sensor 1 owing to the temperature change as shown in Fig. 3(b). Moreover, for Sensor 2 with a grating wavelength of $1552.65 \mathrm{~nm}$, the peak wavelength and reflected power are increased as the temperature increases, as shown in Fig. 3(c). This sensing system can be expanded by connecting several sensing heads in parallel to achieve both multipoint and multiparameter monitoring functions for the smart sensing network system.

Both the grating wavelength shift and reflected power versus temperature change are shown in Fig. 4. At the initial temperature $\left(20^{\circ} \mathrm{C}\right)$ with the corresponding peak wavelengths of $1546.58 \mathrm{~nm}$ of Sensor 1 and $1552.65 \mathrm{~nm}$ of Sensor 2, the temperature was increased from 20 to $90{ }^{\circ} \mathrm{C}$ by changing the temperature of the heater in $10{ }^{\circ} \mathrm{C}$ increments. The figure shows that the grating wavelength is redshifted and the reflected power is enhanced as the temperature is increased. The temperature sensitivities of Sensor 1 are about $0.0082 \mathrm{~nm} /{ }^{\circ} \mathrm{C}$ and $0.038 \mathrm{dBm} /{ }^{\circ} \mathrm{C}$, and those of Sensor 2 are about $0.0074 \mathrm{~nm} /{ }^{\circ} \mathrm{C}$ and $0.044 \mathrm{dBm} /{ }^{\circ} \mathrm{C}$. 


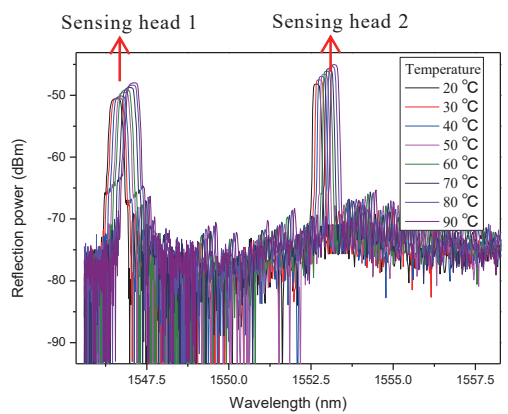

(a)

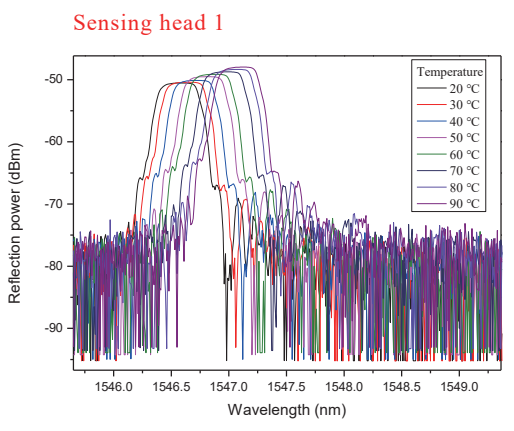

(b)

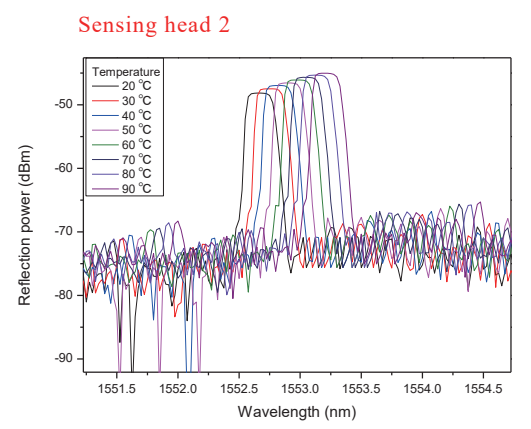

(c)

Fig. 3. (Color online) Measured reflected spectra of two sensing heads for the sensor with the temperature increased from 20 to $90^{\circ} \mathrm{C}$ : (a) temperature measurement from 20 to $90{ }^{\circ} \mathrm{C}$, (b) spectrum of sensing head 1 , and (c) spectrum of sensing head 2 .

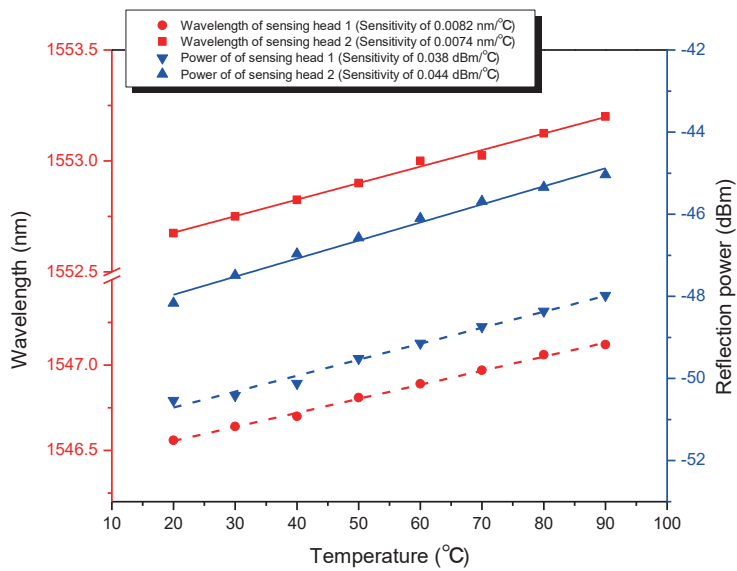

Fig. 4. (Color online) Relationship curves between the reflected power and the wavelength shift with two sensing heads of the sensor with changing temperature and constant stress.

\subsection{Strain}

Figure 5 shows the reflected wavelength spectra for various stress intensities. The curves for the reflected wavelength shift versus the reflected power change are shown in Fig. 6. When the strain increases, the wavelengths of FBG and LPG shift toward longer wavelengths due to 


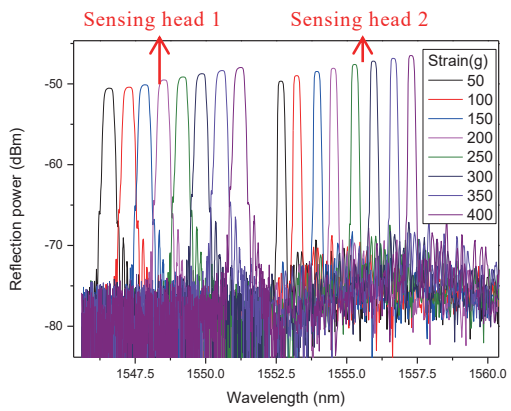

(a)

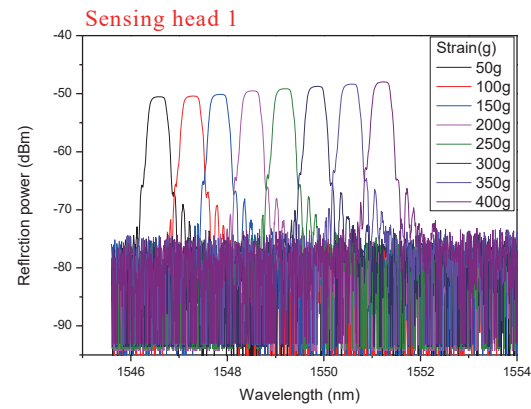

(b)

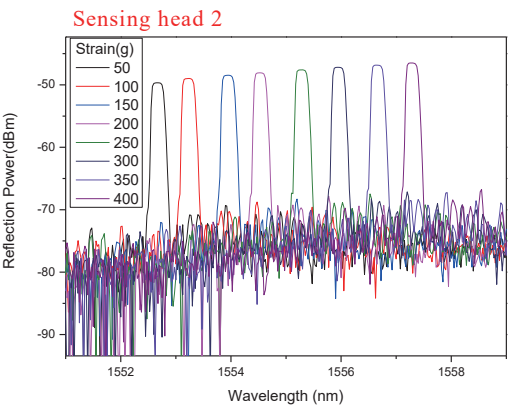

(c)

Fig. 5. (Color online) Reflected spectra of the sensor in a stress range from 50 to 400 g. (a) Stress measurement from 50 to $400 \mathrm{~g}$, (b) spectrum of sensing head 1, and (c) spectrum of sensing head 2.

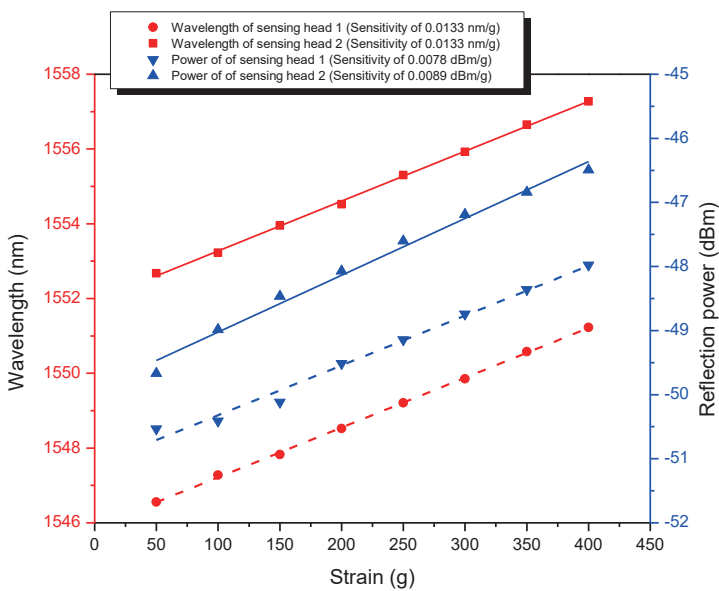

Fig. 6. (Color online) Relationship curves between the reflected power and the wavelength shifts of two sensing heads in a stress range from 50 to $400 \mathrm{~g}$ at a temperature of $20^{\circ} \mathrm{C}$.

increments in the grating period and the effective refractive index. According to Figs. 5 and 6, when the stress intensity is increased from 50 to $100 \mathrm{~g}$, the reflected peak wavelength shift of Sensor 1 is from 1546.55 to $1547.27 \mathrm{~nm}$ corresponding to a total wavelength shift of $0.72 \mathrm{~nm}$, and the reflected power changes from -49.669 to $-48.986 \mathrm{dBm}$ corresponding to a total power variation of $0.683 \mathrm{dBm}$. The sensitivities to stress are about $0.0133 \mathrm{~nm} / \mathrm{g}$ and $0.0078 \mathrm{dBm} / \mathrm{g}$ for Sensor 1 and $0.0133 \mathrm{~nm} / \mathrm{g}$ and $0.0089 \mathrm{dBm} / \mathrm{g}$ for Sensor 2 . 


\subsection{Summary}

From the experimental results, we can obtain the sensitivity of each sensing head for temperature and stress to compose a matrix to determine the temperature $(T)$ and stress $(S)$ changes. A transfer matrix can be expressed as

$$
\left[\begin{array}{l}
\Delta T \\
\Delta S
\end{array}\right]=\left[\begin{array}{ll}
M_{11} & M_{12} \\
M_{21} & M_{22}
\end{array}\right]^{-1}\left[\begin{array}{l}
\Delta \lambda \\
\Delta P
\end{array}\right]
$$

The coefficients of $M_{11}, M_{12}, M_{21}$ and $M_{22}$ (Table 1) can be found from the wavelength shift and reflectivity power change of the sensors in a separate experiment using various temperatures and stresses applied to the fiber grating. Furthermore, we can observe the wavelength shift and power change from the OSA and substitute it into this matrix to obtain the data for both temperature and stress.

In this research, the sensor can be used to measure the temperature and stress variation simultaneously. The sensor experimentally demonstrated the capability of measuring the two physical parameters of temperature and stress as shown in Fig. 7. According to this figure, the coefficients of the transfer matrix can be determined by the wavelength shift and reflectivity change of the loss dips in a separate experiment at various pressures and temperatures applied to the sensor. For the given coefficients of the transfer matrix, the pressure and temperature changes can be determined by solving an equation. Thus, the experimental results have confirmed that this sensor can measure two parameters simultaneously.

Table 1

Coefficients $M_{11}, M_{12}, M_{21}$, and $M_{22}$ for Sensors 1 and 2.

\begin{tabular}{lcccc}
\hline & $M_{11}\left(\mathrm{~nm} /{ }^{\circ} \mathrm{C}\right)$ & $M_{12}(\mathrm{~nm} / \mathrm{g})$ & $M_{21}\left(\mathrm{dBm} /{ }^{\circ} \mathrm{C}\right)$ & $M_{22}(\mathrm{dBm} / \mathrm{g})$ \\
\hline Sensor 1 & 0.0082 & 0.0133 & 0.038 & 0.0078 \\
Sensor 2 & 0.0074 & 0.0133 & 0.044 & 0.0089 \\
\hline
\end{tabular}

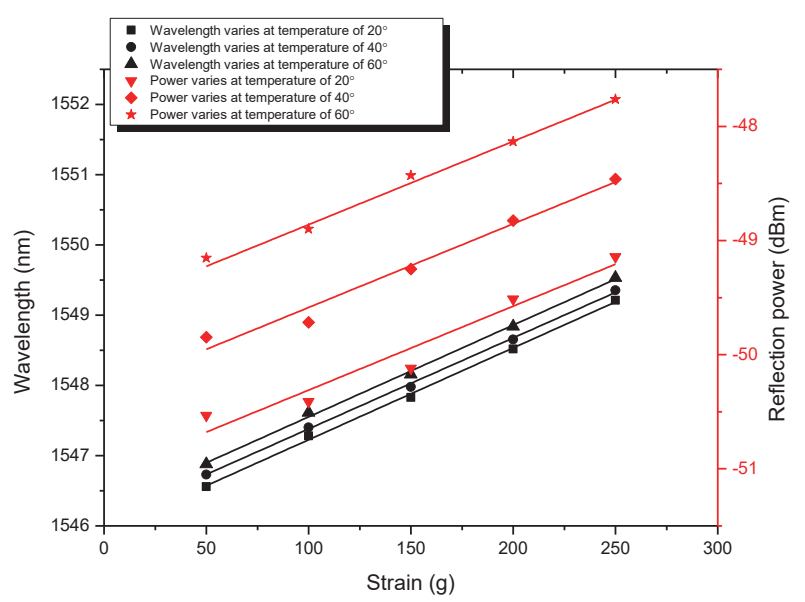

Fig. 7. (Color online) The sensor has experimentally demonstrated the capability of measuring two physical parameters. 


\section{Conclusions}

In this paper, we proposed a new sensing head based on an LPG concatenated with an FBG for monitoring remote temperature and stress simultaneously. Using the transfer matrix, the measured reflected wavelength shift and reflected power variation of the sensor could be utilized for measuring temperature and stress. Therefore, the proposed scheme of an LPG concatenated with an FBG is a highly promising and simple design for a wide range of sensing applications. This sensor can practically be used for real-time monitoring of strain and temperature simultaneously. The temperature sensitivities are about $0.0082 \mathrm{~nm} /{ }^{\circ} \mathrm{C}$ and $0.044 \mathrm{dBm} /{ }^{\circ} \mathrm{C}$, and the strain sensitivities are about $0.0133 \mathrm{~nm} / \mathrm{g}$ and $0.0089 \mathrm{dBm} / \mathrm{g}$. In the future, the sensitivity of this sensor can be improved by adjusting the packaging or using different types of fibers for a wide range of applications.

\section{Acknowledgments}

The authors would like to specifically thank the Ministry of Science and Technology, Taiwan, for sponsoring this research under Contract Nos. MOST 103-2221-E-035-006-MY3 and MOST 105-2622-E-035-015-CC3.

\section{References}

1 H. J. Patrick, G. M. Williams, A. D. Kersey, J. R. Pedrazzani, and A. M. Vengsarkar: Photonics Technol. Lett. 8 (1996) 1223.

2 M. G. Xu, J. L. Archambault, L. Reekie, and J. P. Dakin: Electronics Lett. 30 (1994) 1085.

3 W. C. Du, X. M. Tao, and H. Y. Tam: Photonics Technol. Lett. 11 (1999) 105.

4 N. Liu, Y. Li, Y. Wang, H. Wang, W. Liang, and P. Lu: Opt. Express 19 (2011) 13880.

5 J. Du and Z. He: Opt. Express 21 (2013) 27111.

6 A. M. Vengsarlar, P. J. Lemaire, J. B. Judkins, Vikram Bhatia, T. Erdogan, and J. E. Sipe: J. Lightwave Technol. 14 (1996) 58.

7 A. D. Kersey, M. A. Davis, H. J. Patrick, M. LeBlanc, K. P. Koo, C. G. Askins, M. A. Putnam, and E. J. Friebele: J. Lightwave Technol. 15 (1997) 442.

8 V. Bhatia and A. M. Vengsarkar: Optics Lett. 21 (1996) 692. 